\title{
Avaliação da Qualidade de Vida e Prevalência de Sintomas Depressivos em Pacientes Oncológicos Submetidos à Radioterapia
}

doi: https://doi.org/10.32635/2176-9745.RBC.2020v66n1.775

\author{
Assessment of Quality of Life and Prevalence of Depressive Symptoms in Oncologic Patients submitted to Radiotherapy \\ Evaluación de la Calidad de Vida y Prevalencia de Síntomas Depresivos en Radioterapia en Pacientes Oncológicos
}

\author{
Antonio Augusto Claudio Pereira'; Nayara de Paula Passarin²; Jordana Henriques Coimbra ${ }^{3}$; Gabriela Grasso Pacheco ${ }^{4}$; Marcel \\ Pereira Rangel ${ }^{5}$
}

Resumo

Introduçáo: A qualidade de vida de pacientes oncológicos tem sido objeto de estudo em muitos trabalhos brasileiros. Contudo, apesar da alta prevalência de indivíduos submetidos à radioterapia, poucos estudos com ênfase nesse grupo de pacientes foram identificados. Objetivo: Avaliar a qualidade de vida e a prevalência de sintomas depressivos em pacientes com neoplasias malignas durante o tratamento radioterápico. Método: Estudo transversal quantitativo realizado com 153 pacientes oncológicos em vigência de tratamento radioterápico em um centro especializado em oncologia e radioterapia, localizado no Noroeste do Estado do Paraná. Os dados foram coletados entre março e setembro de 2018. O European Organization for Research and Treatment of Cancer Quality of Life Questionnaire (EORTC QLQ-C30) e o Inventário de Depressáo de Beck foram utilizados para avaliar a qualidade de vida e os sintomas depressivos, respectivamente. Resultados: Os domínios "qualidade de vida", "funçâo cognitiva" e "função social” foram os que menos se mostraram prejudicados na amostra estudada, enquanto "insônia", "perda de apetite" e "dificuldades financeiras" destacaram-se entre os maiores preditores de baixa qualidade de vida. Ademais, contatou-se que $22 \%$ dos indivíduos avaliados apresentaram algum grau de transtorno de humor, sendo $11 \%$ diagnosticados com depressão. Conclusáo: $\mathrm{O}$ declínio na qualidade de vida e a prevalência de sintomas depressivos em pacientes oncológicos, mesmo os em vigência de radioterapia, enaltecem a importância de intervenções precoces que visem a restabelecer a funcionalidade e o bem-estar. Palavras-chave: Neoplasias/radioterapia; Neoplasias/psicologia; Depressão; Qualidade de Vida; Assistência à Saúde.

\section{Abstract}

Introduction: The quality of life of cancer patients has been studied in many Brazilian papers. However, despite the high prevalence of individuals undergoing radiotherapy, few studies with emphasis in this group of patients have been identified. Objective: To evaluate the quality of life and the prevalence of depressive symptoms in patients with malignant neoplasms undergoing radiotherapy treatment. Method: Quantitative cross-sectional study with 153 cancer patients undergoing radiotherapy treatment at an oncology and radiotherapy specialized center, located in the northwest of Paraná state. Data were collected between March and September 2018. The European Organization for Research and Treatment of Cancer Quality of Life Questionnaire (EORTC QLQ-C30) and the Beck Inventory were used to assess quality of life and depressive symptoms, respectively. Results: The domains "quality of life", "cognitive function" and "social function" were the least affected in the studied sample, while "insomnia", "loss of appetite" and "financial difficulties" stood out among the higher predictors of poor quality of life. In addition, it was found that $22 \%$ of the individuals evaluated had some degree of mood disorder, $11 \%$ being diagnosed with depression. Conclusion: The decline in quality of life and the prevalence of depressive symptoms in cancer patients, even those undergoing radiotherapy, emphasize the importance of early interventions aimed at restoring functionality and well-being.

Key words: Neoplasms/radiotherapy; Neoplasms/psychology; Depression; Quality of Life; Delivery of Health Care.

\section{Resumen}

Introducción: La calidad de vida de los pacientes con cáncer se ha estudiado en muchos estudios brasileńos. Sin embargo, a pesar de la alta prevalencia de individuos sometidos a radioterapia, se han identificado pocos estudios con énfasis en este grupo de pacientes. Objetivo: Evaluar la calidad de vida y la prevalencia de síntomas depresivos en pacientes con neoplasias malignas sometidas a radioterapia. Método: Estudio transversal cuantitativo realizado con 153 pacientes con cáncer sometidos a tratamiento de radioterapia en un centro especializado en oncología y radioterapia, ubicado en el Noroeste del Estado de Paraná. Los datos se recopilaron entre marzo y septiembre de 2018. El European Organization for Research and Treatment of Cancer Quality of Life Questionnaire (EORTC QLQ-C30) y el Inventario Beck se utilizaron para evaluar la calidad de vida y los síntomas depresivos, respectivamente. Resultados: Los dominios "calidad de vida", "función cognitiva" y "función social" fueron los menos afectados en la muestra estudiada, mientras que el "insomnio", la "pérdida de apetito" y las "dificultades financieras" se destacaron entre los dominios. predictores más altos de mala calidad de vida. Además, se encontró que el $22 \%$ de los individuos evaluados tenían algún grado de trastorno del estado de ánimo, y el 11\% fue diagnosticado con depresión. Conclusión: La disminución de la calidad de vida y la prevalencia de síntomas depresivos en pacientes con cáncer, incluso en aquellos que reciben radioterapia, enfatizan la importancia de las intervenciones tempranas destinadas a restaurar la funcionalidad y el bienestar.

Palabras clave: Neoplasias/radioterapia; Neoplasias/psicología; Depresión; Calidad de Vida; Prestación de Atención de Salud.

\footnotetext{
${ }^{1}$ Centro Universitário de Maringá (UniCesumar). Maringá (PR), Brasil. Orcid iD: https://orcid.org/0000-0001-9808-8052

${ }^{2}$ UniCesumar. Maringá (PR), Brasil. Orcid iD: https://orcid.org/0000-0002-0082-438X

${ }^{3}$ UniCesumar. Maringá (PR), Brasil. Orcid iD: https://orcid.org/0000-0001-5568-9317

${ }^{4}$ UniCesumar. Maringá (PR), Brasil. Orcid iD: https://orcid.org/0000-0001-8564-3432

${ }^{5}$ Universidade Estadual de Maringá (UEM). UniCesumar. Maringá (PR), Brasil. Orcid iD: https://orcid.org/0000-0001-9322-8538

Endereço para correspondência: Nayara de Paula Passarin. Av. Londrina, 934 - Zona 8. Maringá (PR), Brasil. CEP 87050-390. E-mail: antonio-augusto_@hotmail.com
} 


\section{INTRODUÇÃO}

Neoplasias malignas são caracterizadas pelo crescimento celular desordenado, rápido e invasivo de tecidos e órgãos, e constituem a segunda principal causa de morte em todo o mundo, sendo responsáveis por cerca de 9,6 milhôes de óbitos em 2018, atrás apenas das doenças cardiovasculares ${ }^{1}$. Entre os tumores mais incidentes sobre homens brasileiros, estão os cânceres de próstata, vias respiratórias inferiores, pulmão, cólon e reto. Já entre as mulheres, o câncer de mama ocupa a posição de maior destaque, ultrapassando os tumores do trato gastrointestinal e do colo uterino. Cerca de 420 mil casos novos de câncer foram estimados para o país no biênio 2018-2019, excetuando-se as neoplasias de pele não melanoma².

Por ser multicausal e náo distinguir entre sexo ou idade, o câncer pode se manifestar a qualquer momento, de forma insidiosa ou abrupta, com distintas apresentaçôes ${ }^{2,3}$. É notável o avanço nas pesquisas envolvendo desde a biologia tumoral até aspectos preventivos, diagnósticos e terapêuticos, implicando em melhor prognóstico de muitos tumores ${ }^{4,5}$. Entre as abordagens terapêuticas, a radioterapia é uma modalidade crescente e pode ser empregada isoladamente ou em conjunto com a quimioterapia, com finalidade adjuvante, neoadjuvante ou mesmo paliativa. Cerca de metade dos pacientes oncológicos necessita desse tipo de terapia no Brasil. É um recurso de alta demanda, e custo elevado, uma vez que os equipamentos, sua manutenção e profissionais qualificados são onerosos ${ }^{6}$.

No momento de escolher qual terapia utilizar, modalidade e conformação, deve-se levar em conta o estado geral do paciente, o tamanho e localização da tumoração, assim como a aplicabilidade e habilidade no manejo da técnica escolhida. Desse modo, a radioterapia pode ser subdividida em duas técnicas: a teleterapia na qual a radiação é aplicada externamente ao paciente por meio de raios-gama, raios-x e feixes de elétrons; e a braquiterapia na qual a irradiação é introduzida internamente ao tumor ou em suas proximidades ${ }^{7}$. A radioterapia é indolor, e o paciente não detém visibilidade da aplicação dos raios. Entretanto, muitos são os possíveis efeitos adversos, mediatos ou tardios ${ }^{8}$.

A qualidade de vida de pacientes oncológicos tem sido objeto de estudo em muitos trabalhos brasileiros, principalmente quando em vigência de quimioterapia. Entretanto, poucos estudos com ênfase sobre o paciente em tratamento radioterápico foram identificados ${ }^{9,10}$. Ante os desafios enfrentados pelo paciente e familiares, do processo diagnóstico ao terapêutico ${ }^{11-14}$, e em razão do impacto significativo da doença sobre a economia, com mortes prematuras e incapacidades ${ }^{15-17}$, identificar fatores para o declínio do bem-estar dos pacientes pode contribuir para o direcionamento de intervençóes.

$\mathrm{O}$ estresse psicológico e as manifestaçóes mentais podem surgir mesmo antes do diagnóstico, enquanto o paciente é submetido a uma série de exames investigativos, e costumam estender-se durante toda a execução do planejamento terapêutico - que pode ser curto, ou levar muitos anos ${ }^{18,19}$. Além do impacto da própria patologia, com todos os seus estigmas, mudanças na aparência, vida social, atividades diárias básicas, como alimentação, higienização, podem ocorrer, contribuindo negativamente para o status psicológico do paciente ${ }^{20}$. As mudanças comportamentais, tendência a isolamento ou reclusão social, podem inclusive desencadear quadros severos de transtornos ansiosos e/ou depressivos, transitórios ou persistentes, e muitas vezes desapercebidos pelo olhar profissional ou familiar ${ }^{14,21}$.

Nesse sentido, o presente estudo objetivou avaliar a qualidade de vida e a prevalência de sintomas depressivos em pacientes com neoplasias malignas durante o tratamento radioterápico.

\section{MÉTODO}

Este estudo foi aprovado pelo Comitê de Ética em Pesquisa em Seres Humanos do Centro Universitário de Maringá (UniCesumar), CAAE: 81966617.4.0000.5539, sob o n. ${ }^{\circ}$ de parecer 2.197.264, em consonância com a Resolução n.o 466/12 do Conselho Nacional de Saúde.

O período de coleta ocorreu entre os meses de março e setembro de 2018, sendo os pacientes entrevistados pelos pesquisadores durante a espera para sessão de radioterapia, mediante leitura e preenchimento do Termo de Consentimento Livre e Esclarecido pelos participantes. A participação foi voluntária, e os participantes receberam todas as informaçóes referentes aos objetivos do estudo e aos aspectos éticos, tendo sido garantido o anonimato. As informaçôes clínicas dos pacientes foram extraídas dos respectivos prontuários.

Trata-se de um estudo exploratório descritivo com delineamento transversal e abordagem quantitativa. Foram convidados a participar todos pacientes em tratamento radioterápico no Serviço do Centro de Oncologia e Radioterapia Sant'Ana, localizado na cidade de Maringá PR, durante o período de coleta. A amostra foi constituída seguindo os critérios de elegibilidade: idade igual ou superior a 18 anos; e diagnóstico confirmado de neoplasia maligna, sendo, assim, foram incluídos 153 indivíduos. Não houve exclusão de questionários.

O primeiro instrumento é o Inventário de Depressão de Beck, o qual consiste em 21 itens com uma escala de quatro níveis, de 0 a 3 pontos, em que zero implica 
ausência de sintomas e três, presença de sintomas graves. Nele, são avaliados: 1) humor deprimido; 2) pessimismo; 3) sensação de fracasso; 4) perda de satisfação; 5) sentimento de culpa; 6) sensação de punição; 7) autorrancor (ódio, aversão); 8) autoacusação; 9) ideação suicida; 10) choro; 11) irritabilidade; 12) isolamento social; 13) indecisão; 14) imagem corporal alterada; 15) inibição para o trabalho; 16) anormalidade do sono; 17) fatigamento; 18) perda de apetite; 19) perda de peso; 20) preocupaçôes somáticas; e 21) perda de libido. A escala é graduada com afirmaçóes de 0 a 3, onde zero é ausência do sintoma e três, a presença máxima do sintoma. $\mathrm{O}$ escore mínimo é zero e o máximo, 63. Após a aplicação, obteve-se um escore total que é a soma dos 21 itens, considerando as seguintes categorias: (1) ausência de depressão - pontuação de 0 a 15 ; (2) disforia - pontuação de 16 a 20; (3) depressão - pontuação de 21 a 63 . É valido mencionar que esse questionário fora interpretado por um profissional devidamente habilitado.

O segundo instrumento utilizado é o European Organization for Research and Treatment of Cancer Quality of Life Questionnaire (EORTC QLQ-C30). Trata-se de um questionário multidimensional constituído por 30 questôes que pretendem avaliar o domínio físico, psicológico, o nível de independência, o aspeto social e o ambiente circundante (recursos financeiros e vivência no lar) e que se encontram divididas em três escalas e 15 subescalas: 1) o estado global de saúde e qualidade de vida; 2) a escala funcional composta pelo funcionamento físico, limitaçóes funcionais como, por exemplo, conseguir realizar normalmente tarefas diárias, funcionamento emocional, funcionamento cognitivo e funcionamento social; 3) a escala sintomática composta pelas subescalas fadiga, náuseas e vómitos, dor, falta de ar, insónia, falta de apetite, obstipação, diarreia e dificuldades financeiras. Esse instrumento, elaborado pelo grupo EORTC, fora aplicado em sua versão em português, a qual se encontra traduzida e validada, disponível no sítio de Internet dessa organização. As questóes 1 a 28 possuem escala com quatro níveis, enquanto os itens 29 e 30, sete níveis. Esse instrumento permite o cálculo de escores; para tal, estima-se um escore bruto inicial, dado pela média aritmética simples dos respectivos itens de um domínio.

A princípio, foi realizada uma análise descritiva dos resultados para a obtenção de gráficos e tabelas de frequência, com o intuito de caracterizar os indivíduos. Para descrição dos resultados, serão utilizadas a frequência absoluta e a porcentagem para as variáveis categóricas. Para avaliar as relaçóes entre as variáveis de natureza quantitativa medidas nos participantes do estudo, foi utilizado o teste de correlação de Pearson. Todas as análises foram realizadas com o auxílio do ambiente estatístico $\mathrm{R}$ ( $R$ Development Core Team), versão 3.5.

\section{RESULTADOS}

A amostra foi composta 153 indivíduos de ambos os sexos com idade variável (23-98), sendo 40\% dos participantes com idade superior a 60 anos.

A Figura 1 mostra a concentraçáo dos tipos de câncer, em que a moda é o câncer de mama (45,75\%), seguido pelos tipos ginecológicos $(11,11 \%)$ e de cabeça e pescoço (10,46\%). Além disso, 6,54\% das observações não apresentam resposta.

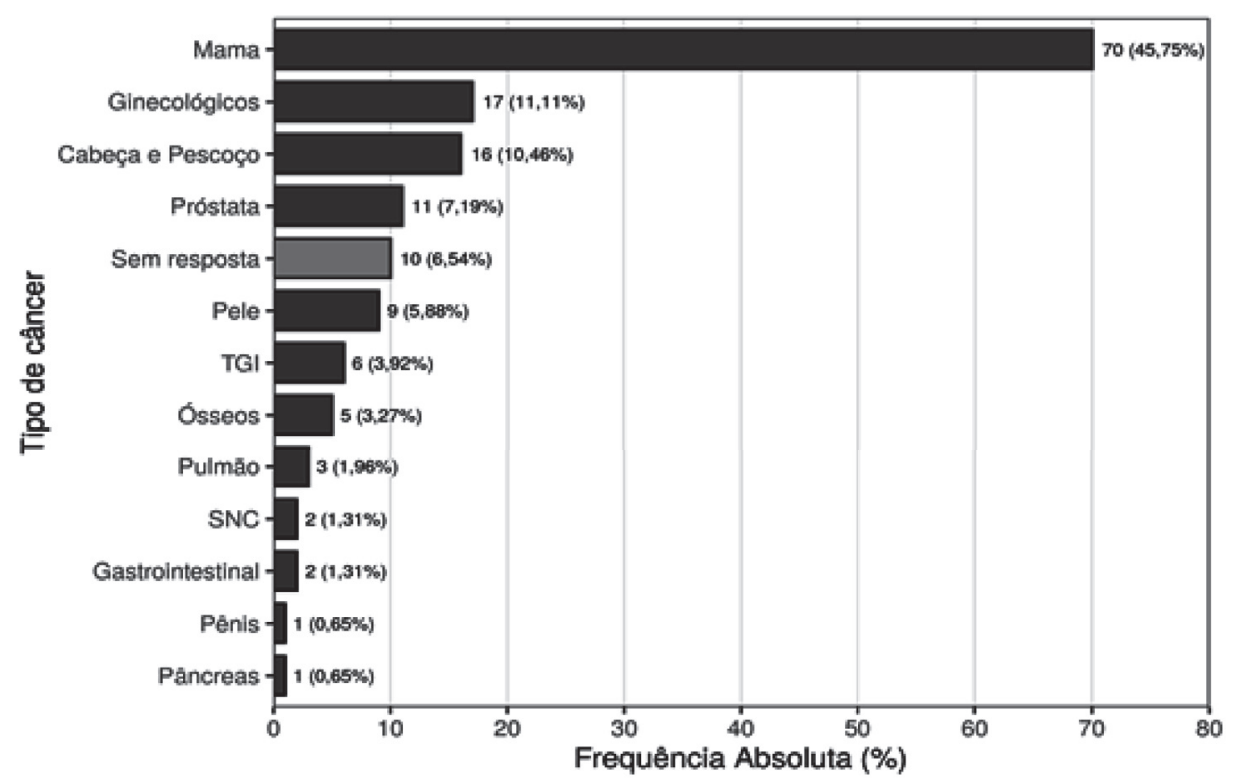

Figura 1. Gráfico de barras da frequência absoluta dos tipos de câncer 
Em relação às modalidades de tratamento empregadas, verificou-se que a maioria dos pacientes do serviço, $52 \%$ (80), foi submetida à radioterapia adjuvante, seguido por $20 \%$ (31) na modalidade curativa. As demais modalidades, como neoadjuvante e paliativa, representaram menos de $10 \% \mathrm{da}$ amostra. Quanto à técnica utilizada, observou-se que a técnica bidimensional 2D é empregada em 55\% (84) da amostra, seguida pela técnica tridimensional conformacional 3D-CTR com 24\% (36). As demais técnicas, radioterapia de intensidade modulada (IMRT) e braquiterapia, representaram menos de $5 \%$ dos participantes. Ainda, 28 prontuários não continham tais informaçôes no campo padrão, provavelmente por estarem em fase de discussão e planejamento terapêutico.

Após a análise univariada, avaliou-se a distribuição dos tipos de câncer em cada modalidade de tratamento, desconsiderando os 28 pacientes que náo tiveram resposta em pelo menos uma das variáveis dessa modalidade de tratamento, totalizando 125 pacientes (Tabela 1).
Observa-se que quase metade dos pacientes (46,67\%) com o câncer de cabeça e pescoço foi submetida à modalidade curativa, enquanto nenhum, à neoadjuvante. Em termos dos tumores ginecológicos, duas modalidades são predominantes, adjuvante $(47,06 \%)$ e curativa $(41,18 \%)$.

Ao se avaliar o tipo de câncer de mama, nota-se que quase todos $(92,98 \%)$ pertencem à modalidade adjuvante, e nenhum à modalidade curativa. A maioria $(62,50 \%)$ dos pacientes com neoplasias de pele estava em adjuvância. Para o câncer próstata, a maior parte $(81,82 \%)$ dos pacientes foi submetida à modalidade curativa.

Considerando os dados obtidos por meio do instrumento EORTC QLC-C30, expressos na Figura 2, no qual foi avaliada a distribuição dos escores em funçấo dos domínios, nota-se que os domínios funcionais e de qualidade de vida apresentam alta concentração na maior categoria de escore (maior que 90), com destaque para as funçóes cognitiva e social, em que $50 \%$

Tabela 1. Frequência relativa (absoluta) das modalidades, por tipo de câncer

\begin{tabular}{c|c|c|c|c|c|c}
\hline Modalidade & $\begin{array}{c}\text { Cabeça e } \\
\text { Pescoço }\end{array}$ & Ginecológicos & Mama & Pele & Próstata & Outro \\
\hline Adjuvante & $33,33 \%(5)$ & $47,06 \%(8)$ & $92,98 \%(53)$ & $62,50 \%(5)$ & $9,09 \%(1)$ & $47,06 \%(8)$ \\
Curativa & $46,67 \%(7)$ & $41,18 \%(7)$ & - & $37,50 \%(3)$ & $81,82 \%(9)$ & $29,41 \%(5)$ \\
Neoadjuvante & - & $5,88 \%(1)$ & $3,51 \%(2)$ & - & - & $5,88 \%(1)$ \\
Paliativa & $20,00 \%(3)$ & $5,88 \%(1)$ & $3,51 \%(2)$ & - & $9,09 \%(1)$ & $17,65 \%(3)$ \\
\hline Total & $100 \%(15)$ & $100 \%(17)$ & $100 \%(57)$ & $100 \%(8)$ & $100 \%(11)$ & $100 \%(17)$ \\
\hline
\end{tabular}

\begin{tabular}{|c|c|c|c|c|c|c|c|c|c|c|}
\hline Qualidade de vida - & 1,99 & 1,99 & 0,66 & 4,64 & 16,56 & 5,96 & 9,27 & 10,6 & 13,91 & $\mathbf{3 4 , 4 4}$ \\
\hline Desempenho fisico- & 1,32 & 0 & 1,32 & 5,3 & 3,97 & 11,92 & 3,97 & 19,87 & 15,89 & 36,42 \\
\hline Desempenho funcional - & 6,04 & 6,71 & 0 & 8,72 & 10,07 & 0 & 17,45 & 0 & 14,77 & 36,24 \\
\hline Funçāo emocional - & 2,67 & 3,33 & 3,33 & 2 & 16 & 8 & 7,33 & 8,67 & 12 & 36,67 \\
\hline Funçăo cognitiva - & 0,67 & 4 & 0 & 7,33 & 6,67 & 0 & 12 & 0 & 19,33 & 50 \\
\hline Função social & 4,7 & 4,03 & 0 & 2,68 & 8,05 & 0 & 16,78 & 0 & 14,09 & 49,66 \\
\hline Fadiga & 26,67 & 10 & 19,33 & 14 & 9,33 & 5,33 & 8,67 & 1,33 & 2 & 3,33 \\
\hline Náusea e vômitos & 56,38 & 14,77 & 0 & 9,4 & 8,72 & 0 & 4,03 & 0 & 2,68 & 4,03 \\
\hline Dor & 40 & 21,33 & 0 & 13,33 & 10 & 0 & 4 & 0 & 6 & 5,33 \\
\hline Dispneia & 83,22 & 0 & 0 & 10,07 & 0 & 0 & 3,36 & 0 & 0 & 3,36 \\
\hline Insônia - & 54,36 & 0 & 0 & 24,16 & 0 & 0 & 9,4 & 0 & 0 & 12,08 \\
\hline Perda de apetite & 65,7 & 0 & 0 & 22,15 & 0 & 0 & 10,07 & 0 & 0 & 12,08 \\
\hline Constipaçăa - & 66,67 & 0 & 0 & 15,33 & 0 & 0 & 10 & 0 & 0 & 8 \\
\hline Diarreia - & 71,14 & 0 & 0 & 20,13 & 0 & 0 & 4,7 & 0 & 0 & 4,03 \\
\hline \multirow[t]{2}{*}{ Dificuldades financeiras - } & 56,76 & 0 & 0 & 22,3 & 0 & 0 & 10,14 & 0 & 0 & 10,81 \\
\hline & {$[0,10]$} & $(10,20]$ & $(20,30]$ & $(30,40]$ & $\begin{array}{c}(40,50] \\
\text { Esc }\end{array}$ & $\begin{array}{l}(50,60] \\
\text { ore }\end{array}$ & $(60,70]$ & $(70,80]$ & $(80,90]$ & $(90,100]$ \\
\hline
\end{tabular}

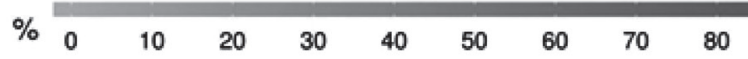

Figura 2. Mapa de calor do percentual de respostas entre escore calculado e dos domínios do instrumento EORTC 
e 49,66\% dos respondentes estão na maior categoria, respectivamente. Os demais domínios apresentam justamente uma maior concentração na menor categoria do escore, sendo que a insônia, a perda de apetite e as dificuldades financeiras possuem mais de $10 \%$ de respostas com escore superior a 90.

Os resultados do Inventário de Depressão de Beck estão expostos na Figura 3 com 21 itens ordenados por aqueles com maior gravidade. A perda de libido apresenta quase $40 \%$ de respostas graves (bastante ou muito), seguida por perda de peso e inibição para o trabalho. Já em termos dos itens cuja gravidade menor (náo ou pouco) representa a grande maioria das respostas, destaca-se ideação suicida e autorrancor que apresentam somente $1 \%$ das respostas com níveis graves.

Posteriormente, faz-se a análise das categorias provenientes da pontuaçáo total do instrumento. Nota-se que a maioria dos respondentes, 78\% (119), foi categorizada com a ausência de depressão, enquanto $11 \%$ (17) com disforia e o restante 11\% (17) com depressáo.

Por fim, avalia-se como se dá a relação entre os instrumentos, utilizando a correlaçáo entre a pontuação final do Inventário de Depressão de Beck com o escore de cada domínio do instrumento EORTC. Observa-se que as correlaçôes com os domínios funcionais e de qualidade de vida são negativas; isto é, quanto maior o escore do Inventário de Depressáo de Beck, menor o seu escore, sendo que função emocional apresenta a correlação linear negativa mais forte. $\mathrm{O}$ contrário acontece para os domínios relativos a sintomas, nos quais a correlação linear é positiva; ou seja, quanto maior a pontuação final do Inventário de Depressão de Beck, maior o escore para esses domínios.

\section{DISCUSSÃO}

Existe uma marcante e multifatorial associação entre o risco de neoplasias malignas e o envelhecimento, na qual, para a maior parte dos cânceres conhecidos, suas incidências e taxas de mortalidade aumentam com o avançar da idade ${ }^{22}$. Os dados encontrados no presente estudo, todavia, contrapóem tal afirmaçáo, na medida em que houve predominância da faixa etária inferior a 60 anos na amostra avaliada, o que chama a atenção para o alto índice de neoplasias malignas em indivíduos jovens nessa população.

A diminuição da qualidade de vida entre pacientes oncológicos pode estar vinculada a diversas condiçóes, como mudanças físicas e emocionais, dor, dependência de terceiros, perda da autoestima, e ao estigma culturalmente atrelado a essa morbidade ${ }^{23}$. A prevalência de transtorno depressivo no âmbito de pacientes oncológicos é maior do que aquela encontrada na populaçáo geral e essas proporçôes variam expressivamente entre os estudos em razão da escolha de amostras específicas e do uso de métodos de avaliação distintos ${ }^{24}$.

Por meio do instrumento EORTC QLQ-C30, constatou-se elevado escore calculado para o item "qualidade de vida" e para os domínios funcionais, com ressalto para "função cognitiva" e "função social", em

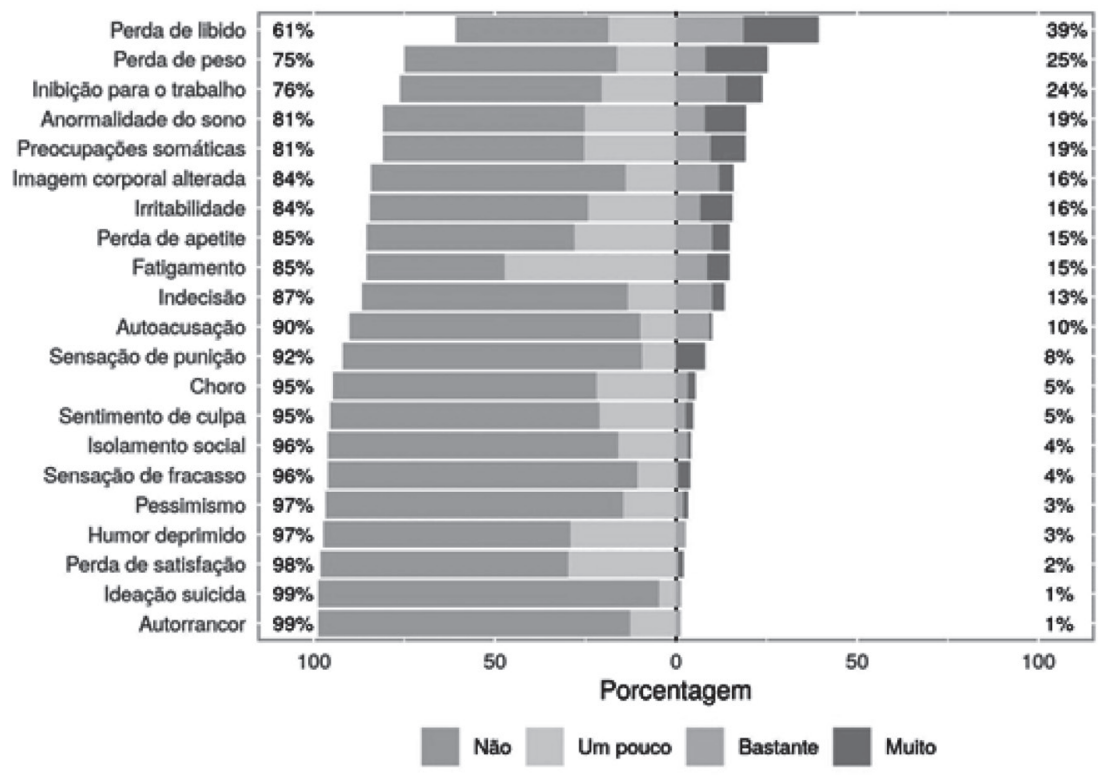

Figura 3. Gráfico de barras do percentual para cada item do Inventário de Depressão de Beck 
que, respectivamente, $50 \%$ e $49,6 \%$ dos participantes obtiveram escores entre 90 e 100, apontando que essas esferas foram as que menos se mostraram prejudicadas nesses pacientes. Em consonância, Faria et al. ${ }^{25}$, em estudo conduzido com 163 pacientes oncológicos, com uso do mesmo instrumento de avaliação, igualmente encontraram os maiores escores no item "qualidade de vida" e nos domínios "função cognitiva" e "função social", com média de 76, 71,6 e 75,9, respectivamente. A boa percepção da qualidade de vida global neste estudo pode estar ligada à qualidade da assistência à saúde recebida por esses indivíduos, bem como ao fato de que somente uma pequena parcela da amostra está em tratamento radioterápico paliativo.

No que concerne à escala de sintomas, os domínios "insônia", "perda de apetite" e "dificuldades financeiras" merecem ser evidenciados por apresentarem mais de 10\% de respostas com escore superior a 90, destacando-se como os maiores preditores de baixa qualidade de vida. No estudo elaborado por Lôbo et al. ${ }^{26}$, com 145 mulheres em tratamento quimioterápico para o câncer de mama, os maiores escores nas escalas de sintomas foram obtidos em "insônia", "fadiga" e "perda de apetite", com médias de 37,93, 36,01 e 33,56, respectivamente. Em estudo semelhante, Mendez et al. ${ }^{27}$ observaram que o único ponto que registrou uma significativa diferença entre os escores antes e após o início do tratamento foi o domínio "dor", para o qual houve queda da mediana de 66 para 33 após dois meses de tratamento. Esses dados permitem inferir que a instituição da modalidade correta de tratamento ao paciente oncológico pode, reconhecidamente, melhorar sua qualidade de vida. Em nosso estudo, 15,3\% dos pacientes obtiveram escore calculado $\geq 50$ para o domínio "dor", ressaltando a emergente necessidade de melhora no controle da dor nesses pacientes.

É sabido que o diagnóstico de câncer e o seu tratamento podem acentuar problemas de sono preexistentes e precipitar novos, como a insônia que, sem tratamento adequado, usualmente se torna crônica e se concatena com o aparecimento de fadiga e depressáo ${ }^{28}$. A perda de apetite, por sua vez, é um sintoma comum entre pacientes oncológicos e atinge cerca de $15 \%$ a $20 \%$ deles, sendo decorrente de alteração no equilíbrio de neurotransmissores estimulantes e inibitórios do apetite, e é induzida por hormônios e citocinas inflamatórias que atingem o núcleo arqueado do hipotálamo ${ }^{29}$.

A partir do Inventário de Depressão de Beck, constatou-se que $22 \%$ dos indivíduos avaliados apresentaram algum grau de alteraçáo do humor, sendo $11 \%$ diagnosticados com transtorno depressivo maior. Na literatura, observam-se taxas ainda maiores, como o referido por Tsaras et al..$^{30}$ em estudo com 152 pacientes diagnosticadas com câncer de mama, cuja prevalência de depressáo foi de $38,2 \%$. Mostrando expressiva semelhança com os dados encontrados no presente estudo, Wagner et al. ${ }^{31}$, em seu estudo com 455 pacientes em tratamento radioterápico, também sendo a maioria acometida por câncer de mama, encontraram uma taxa de $10,1 \%$ de depressão. $\mathrm{O}$ diagnóstico de depressão em pacientes oncológicos é visto como um desafio, uma vez que os sintomas relacionados à neoplasia per se, os efeitos colaterais de fármacos e os sintomas depressivos propriamente ditos podem estar sobrepostos $^{32}$.

No tocante à depressão em pacientes submetidos especificamente à radioterapia, Panwar et al..$^{33}$ realizaram ensaio clínico randomizado, duplo-cego, placebo controlado com pacientes sem depressáo que estavam prestes a se submeter ao tratamento com radioterapia. No grupo placebo, 39\% dos indivíduos que receberam radiação como parte inicial do tratamento desenvolveram depressão durante o período do estudo, em comparação com apenas $12 \%$ dos pacientes que não receberam radioterapia nessa ocasiáo. Em estudo anterior ${ }^{34}$, a populaçáo que se submeteu à radioterapia como terapia inicial apresentou, igualmente, uma probabilidade significativamente maior de desenvolver depressão do que os pacientes tratados cirurgicamente. Essa forte associação entre a depressão e a realização de radioterapia como parte do tratamento pode ser explicada pelo alto índice de efeitos colaterais causados por essa modalidade terapêutica, tais como náuseas, vômitos, cefaleia, alopecia, disfagia, xerostomia, perda temporária do paladar, inapetência, sensação de fraqueza, diarreias e cólicas, sendo a intensidade dos sintomas variáveis conforme o local da radiação ${ }^{35}$.

Neste estudo, os itens que receberam a maior porcentagem de respostas graves no Inventário de Depressão de Beck foram perda de libido, perda de peso e inibiçáo para o trabalho. A perda de peso pode estar relacionada à caquexia comumente desenvolvida por pacientes com câncer, resultante de alteraçôes no metabolismo energético e do turnover de proteínas musculares $^{36}$. Por sua vez, a presença de uma doença debilitante, o uso de medicamentos que inibem a libido, cirurgias e estados emocionais negativos, muitas vezes em concomitância, justificam a alta prevalência da perda de libido em pacientes oncológicos ${ }^{37}$.

\section{CONCLUSÃO}

Este estudo objetivou avaliar a qualidade de vida e a prevalência de sintomas depressivos dos pacientes com câncer em tratamento radioterápico em um centro especializado em oncologia e radioterapia, localizado no 
Noroeste do Estado do Paraná. São múltiplos os fatores que podem contribuir para a redução da qualidade de vida e o aumento da prevalência de depressáo nessa populaçáo, tais como mudanças físicas, alteraçôes emocionais, sintomas associados à doença e ao seu tratamento, perda da autoestima e perda de libido. Soma-se a isso o difícil diagnóstico de depressão em pacientes com câncer, já que sintomas da própria neoplasia e aqueles oriundos do tratamento farmacológico podem se sobrepor ao transtorno depressivo.

Verificou-se, por meio do instrumento EORTC QLQ-C30, que os domínios "qualidade de vida", "função cognitiva" e "função social" foram os que menos se mostraram prejudicados nesses pacientes. $\mathrm{Na}$ escala de sintomas, os domínios "insônia", "perda de apetite" e "dificuldades financeiras" apresentaram mais de 10\% de respostas com escore superior a 90, se destacando como os maiores preditores de baixa qualidade de vida.

A partir do Inventário de Depressáo de Beck, contatou-se que $22 \%$ dos indivíduos avaliados apresentaram algum grau de transtorno de humor, sendo $11 \%$ diagnosticados com depressão, e os itens que receberam a maior porcentagem de respostas graves foram perda de libido, perda de peso e inibição para o trabalho.

Uma vez que a baixa qualidade de vida e os maiores índices de depressão são realidade entre os pacientes oncológicos, conforme evidenciado neste e em outros estudos, faz-se necessário o rastreamento desses indivíduos mediante escores apropriados, visando ao diagnóstico e à intervençáo precoces para o restabelecimento de funcionalidade e bem-estar. Novos estudos são necessários para estimar precisamente qual a prevalência dessas comorbidades, indicar quais são os fatores associados a elas e elaborar um plano terapêutico, medicamentoso ou não, passível de ser aplicado nessa população.

\section{CONTRIBUIÇÕES}

Antonio Augusto Claudio Pereira, Nayara de Paula Passarin e Marcel Pereira Rangel participaram da concepção e desenho do trabalho; aquisição, análise, interpretação dos dados da pesquisa; redação e revisão crítica com contribuição intelectual. Jordana Henriques Coimbra participou da concepção e desenho do trabalho; aquisição, análise e interpretação dos dados da pesquisa. Gabriela Grasso Pereira participou da redação e revisão crítica com contribuiçáo intelectual. Todos os autores aprovaram a versão final a ser publicada.

\section{DECLARAÇÃO DE CONFLITO DE INTERESSES}

Nada a declarar.

\section{FONTES DE FINANCIAMENTO}

Não há.

\section{REFERÊNCIAS}

1. Word Health Organization. Cancer [Internet]. Genève: WHO; [date unknown]. Cancer: key facts. [updated 2018 Sept 12; cited 2019 May 23]. Available from: https://www.who.int/en/news-room/fact-sheets/detail/ cancer

2. Instituto Nacional de Câncer Jóse de Alencar Gomes da Silva. Estimativa 2018: incidência de câncer no Brasil. Rio de Janeiro: INCA; 2017.

3. Oliveira MM, Malta DC, Guauche H, et al. Estimativa de pessoas com diagnóstico de câncer no Brasil: dados da Pesquisa Nacional de Saúde, 2013. Rev Bras Epidemiol. 2015;18(2):146-57. doi: https://doi.org/10.1590/19805497201500060013

4. Secoli SR, Padilha KG, Leite RCBO. Avanços tecnológicos em oncologia: reflexóes para a prática de enfermagem. Rev Bras Cancerol. 2005;51(4):331-7.

5. Ferrão ARC. O tratamento de metástases ósseas de fração única vs múltiplas fraçôes com 3D CRT ou SBRT: artigo de revisão sistemática. Saúde Tecnol. 2015;(14):36-41. doi: http://dx.doi.org/10.25758/set.1254

6. Araújo LP, Sá NM, Atty ATM. Necessidades atuais de radioterapia no SUS e estimativas para o ano de 2030. Rev Bras Cancerol. 2016;62(1):35-42

7. Instituto Nacional de Câncer. Atualização para técnicos em radioterapia. Rio de Janeiro: INCA; 2010.

8. Instituto Nacional de Câncer. Açóes de enfermagem para o controle do câncer: uma proposta de integração ensino-serviço. 3. ed. atual. amp. Rio de Janeiro: INCA; 2008.

9. Xiao C, Miller AH, Felger J, et al. A prospective study of quality of life in breast cancer patients undergoing radiation therapy. Adv Radiat Oncol. 2016;1(1):10-6. doi: http://dx.doi.org/10.1016/j.adro.2016.01.003

10. Movsas B, Hu C, Sloan J, et al. Quality of life analysis of a radiation dose-escalation study of patients with non-small-cell lung cancer: a secondary analysis of the radiation therapy oncology group 0617 randomized clinical trial. JAMA Oncol. 2016;2(3):359-67. doi: http://dx.doi.org/10.1001/jamaoncol.2015.3969

11. Ferreira AS, Bicalho BP, Oda JMM, et al. Câncer de mama: estimativa da prevalência de ansiedade e depressão em pacientes em tratamento ambulatorial. Arq Ciênc Saúde UNIPAR. 2015;19(3):185-9. doi: https://doi. org/10.25110/arqsaude.v19i3.2015.5548

12. Parker PA, Davis JW, Latini DM, et al. Relationship between illness uncertainty, anxiety, fear of progression and quality of life in men with favourable-risk prostate cancer undergoing active surveillance. BJU Int. 
2016;117(3):469-77. doi: https://doi.org/10.1111/ bju. 13099

13. Castro EK, Romeiro FB, Lima NB, et al. Percepçáo da doença, indicadores de ansiedade e depressão em mulheres com câncer. Psic Saúde Doença. 2015;16(3):359-72. doi: http://dx.doi.org/10.15309/15psd160307

14. Seemann T, Pozzobom F, Vieira MCS, et al. Influência de sintomas depressivos na qualidade de vida em homens diagnosticados com câncer de próstata. Rev Bras Geriatr Gerontol. 2018;21(1):72-81. doi: https:// doi.org/10.1590/1981-22562018021.170114

15. Malta DC, Silva MMA da. As doenças e agravos não transmissíveis, o desafio contemporâneo na Saúde Pública. Cien Saude Colet. 2018;23(5):1350. doi: https://doi.org/10.1590/1413-81232018235.31552017

16. Silva SS, Aquino TAA, Santos RM. O paciente com câncer: cognições e emoções a partir do diagnóstico. Rev Bras Ter Cogn. 2008;4(2):73-88. doi: https://doi. org/10.5935/1808-5687.20080016

17. Baratto CC, Moraes NA, Wottrich SH. Câncer e imagem corporal: possíveis relaçóes. Rev Context Saúde. 2011;11(20):789-94. doi: https://doi. org/10.21527/2176-7114.2011.20.789-794

18. Cormanique TF, Almeida LEDF, Rech CA, et al. Estresse psicológico crônico e seu impacto no desenvolvimento de neoplasia mamária agressiva. Einstein. 2015;13(3):352-6. doi: https://doi.org/10.1590/S1679-45082015AO3344

19. Botelho ASC, Pereira MG. Qualidade de vida, otimismo, enfrentamento, morbidade psicológica e estresse familiar em pacientes com câncer colorrectal em quimioterapia. Estud Psicol. 2015;20(1):50-60. doi: https://doi. org/10.5935/1678-4669.20150007

20. Pereira NAC, Fortes RC. Autoimagem corporal de pacientes com câncer gastrointestinal. Comun Ciênc Saúde. 2015;26(1/2):29-44.

21. Costa VB. Avaliação da qualidade de vida e capacidade funcional de pacientes com câncer antes e após o tratamento quimioterápico [monografia]. Brasília, DF: Universidade de Brasília; 2016.

22. Pedersen JK, Engholm G, Skytthe A, et al. Cancer and aging: epidemiology and methodological challenges. Acta Onlol. 2016;55(Suppl 1):7-12. doi: https://doi.org/10. 3109/0284186X.2015.1114670

23. Meneguin S, Matos TDS, Ferreira MLSM. Percepção de pacientes oncológicos em cuidados paliativos sobre qualidade de vida. Rev Bras Enferm. 2018;71(4):2114-20. doi: http://dx.doi.org/10.1590/0034-7167-2017-0360

24. Ferreira AS, Bicalho BP, Neves LFG, et al. Prevalência de ansiedade e depressão em pacientes oncológicos e identificação de variáveis predisponentes. Rev Bras Cancerol. 2016;62(4):321-8.

25. Faria SO, Simiáo MMR, Alves FA, et al. Estado nutricional e qualidade de vida em indivíduos com câncer assistidos por organização não governamental.
Rev Bras Cancerol. 2019;65(1):e-08103. doi: https:// doi.org/10.32635/2176-9745.RBC.2019v65n1.103

26. Lôbo SA, Fernandes AFC, Almeida PC, et al. Qualidade de vida em mulheres com neoplasias de mama em quimioterapia. Acta Paul Enferm. 2014;27(6):554-9. doi: https://doi.org/10.1590/1982-0194201400090

27. Mendez LC, Raman S, Wan BA, et al. Quality of life in responders after palliative radiation therapy for painful bone metastases using EORTC QLQ-C30 and EORTC QLQ-BM22: results of a Brazilian cohort. Ann Palliat Med. 2017;6(Suppl 1):S65-S70. doi: https://doi. org/10.21037/apm.2017.04.06

28. Zhou ES, Partridge AH, Syrjala KL, et al. Evaluation and treatment of insomnia in adult cancer survivorship programs. J Cancer Surviv. 2018;11(1):74-9. doi: https:// doi.org/10.1007/s11764-016-0564-1

29. Gangadharan A, Choi SE, Hassan A, et al. Protein calorie malnutrition, nutritional intervention and personalized cancer care. Oncotarget. 2017;8(14):24009-30. doi: https://doi.org/10.18632/oncotarget.15103

30. Tsaras K, Papathanasiou IV, Mitsi D, et al. Assessment of depression and anxiety in breast cancer patients: prevalence and associated factors. Asian Pac J Cancer Prev. 2018;19(6):1661-9. doi: https://doi.org/10.22034/ APJCP.2018.19.6.1661

31. Wagner LI, Pugh SL, Small W Jr, et al. Screening for depression in cancer patients receiving radiotherapy: Feasibility and identification of effective tools in the NRG Oncology RTOG 0841 trial. Cancer. 2018;123(3):48593. doi: https://doi.org/10.1002/cncr.29969

32. Saracino RM, Weinberger MI, Roth AJ, et al. Assessing depression in a geriatric cancer population. Psychooncology. 2017;26(10):1484-90. doi: https://doi. org/10.1002/pon.4160

33. Panwar A, Rieke K, Burke WJ, et al. Identification of baseline characteristics associated with development of depression among patients with head and neck cancer: a secondary analysis of a randomized clinical trial. JAMA Otolaryngol Head Neck Surg. 2018;144(11):1004-10. doi: https://doi.org/10.1001/jamaoto.2018.2228

34. Lydiatt WM, Bessette D, Schmid KK, et al. Prevention of depression with escitalopram in patients undergoing treatment for head and neck cancer: randomized, double-blind, placebo-controlled clinical trial. JAMA Otolaryngol Head Neck Surg. 2013;139(7):678-86. doi: https://doi.org/10.1001/jamaoto.2013.3371

35. Paula Júnior W, Zanini DS. Pacientes em radioterapia: um estudo de coping. Psic, Saúde e Doenças. 2012;13(2):480-93.

36. Pin F, Barreto R, Couch ME, et al. Cachexia induced by cancer and chemotherapy yield distinct perturbations to energy metabolism. J Cachexia Sarcopenia Muscle. 2019;10(1):140-54. doi: https://doi.org/10.1002/ jcsm. 12360 
37. Fleury HJ, Pantaroto HSC, Abdo CHN. Sexualidade em oncologia. Diagn Tratamento. 2011;16(2):86-90.

Recebido em 28/11/2019

Aprovado em 27/2/2020 